\title{
Fibrocartilaginous embolic encephalopathy of the cerebellum and brainstem in a cat
}

\author{
Rebecca McBride ${ }^{1, *}$, Helena Rylander ${ }^{2}$ and Douglas Lyman ${ }^{2}$ \\ ${ }^{I}$ The Ohio State University Veterinary Hospital, 601 Vernon L Tharp St, Columbus, OH 43210, USA \\ ${ }^{2}$ Veterinary Medical Teaching Hospital, University of Wisconsin-Madison, 2015 Linden Dr, Madison, WI 53706, \\ USA
}

\begin{abstract}
A 12-year old male castrated Siamese cat was evaluated for a one-day history of mild obtundation and decerebellate rigidity. Neurological examination findings were consistent with multifocal disease including lesions within the right cerebellum and right brainstem. Investigations included hematology, biochemistry, urinalysis, and urine culture. A definitive diagnosis was not achieved clinically and differential diagnoses included vascular disease, neoplasia, and infectious disease. About nine hours after admission, the cat had an episode where it became rigid and then limp. The cat became agonal and went into cardiopulmonary arrest and attempts of resuscitation were unsuccessful. Post-mortem examination revealed evidence of multiple fibrocartilaginous emboli (FCE) within the cerebellum and within meningeal vessels on the ventral aspect of the brainstem. This is the first reported case of fibrocartilaginous embolism causing an encephalopathy in a cat. While this is a rare disease, it is an important differential diagnosis that should be considered in cases of acute encephalopathies in cats.
\end{abstract}

Keywords: Cerebellum, Embolus, Encephalopathy, Feline, Fibrocartilaginous.

\section{Introduction}

Fibrocartilaginous embolism (FCE) is typically an acute, non-painful, non-progressive myelopathy that results from an ischemic or hemorrhagic infarction of the spinal cord (Gandini and Cizinauskas, 2003). Histological examination of the material found within the vasculature of the spinal cord is typically identical to the nucleus pulposus (Gandini and Cizinauskas, 2003). The pathophysiology of FCE is much debated and several hypotheses have been proposed, but the exact mechanism is poorly understood. The most common theory is that the material comes from an intervertebral disc.

FCE within the spinal cord is commonly reported in the dog and occasionally in the cat (Cauzinille, 2000; Gandini and Cizinauskas, 2003; De Risio and Platt, 2010). It has also been reported in several other species including humans, horses, turkeys, and pigs (Taylor et al., 1977; Johnson et al., 1988; Fuentealba et al., 1991; Stedman et al., 1998; AbdelRazek et al., 2016).

There have been eleven reported cases of FCE causing myelopathies in cats, some confirmed on histopathology and some suspected based on clinical and diagnostic findings. These cases reportedly presented as an acute, lateralizing, non-painful myelopathy and lesions were reported within various regions of the spinal cord (Zaki et al., 1976; Turner et al., 1995; Scott and O'Leary, 1996; Abramson et al., 2002; Coradini et al., 2005; Mikszewski et al., 2006;
Barker et al., 2014). FCE causing an encephalopathy has been rarely reported in both human and veterinary medicine and has never been documented in a cat. To date, this is the first case of an FCE causing an encephalopathy in a cat reported.

\section{Case Details}

A 12-year-old, $3.8 \mathrm{~kg}$, neutered male Siamese cat was referred to the University of Wisconsin-Madison, Veterinary Medical Teaching Hospital for acute onset of mild obtundation and cerebellar signs. The owner had found the cat in lateral recumbency and vocalizing earlier that day. Prior to presentation the cat had been treated by the primary veterinarian with LactatedRingers Solution (LRS) (10 ml $/ \mathrm{kg}$ IV), dexamethasone $(0.5 \mathrm{mg} / \mathrm{kg}$ IV), and clindamycin $(1.6 \mathrm{mg} / \mathrm{kg} \quad \mathrm{PO})$ without any improvement. The cat had a prior history of inflammatory bowel disease and had recently been started on oral prednisone every other day (dose unknown).

On presentation the cat was mildly obtunded, hypothermic $\left(99.6^{\circ} \mathrm{F}\right)$, bradycardic $(100 \mathrm{bpm})$, and had tacky mucous membranes. He was non-ambulatory, laterally recumbent, vocalizing, and displaying decerebellate rigidity (extended thoracic limbs, flexed pelvic limbs at the level of the hips) and opisthotonus. Neurologic examination revealed mildly obtunded mentation, reduced menace response in the right eye, reduced palpebral reflex in the right eye, and absent physiologic nystagmus. 
The remainder of cranial nerves were normal. Postural reactions were intermittently absent in the right thoracic and right pelvic limbs. Muscle tone and spinal reflexes were normal and there was no pain elicited on spinal palpation. Other physical examination findings were unremarkable.

Based on findings on neurologic examination, multifocal central nervous system disease including the right cerebellum and right brainstem was suspected. Differential diagnoses for the lesion included vascular disease (hemorrhage, infarct), neoplasia (lymphoma, meningioma, glioma, secondary metastasis), and infectious diseases (fungal, protozoal, viral, bacterial). A complete blood count (CBC) and biochemical profile were performed. Clinicopathological abnormalities included lymphopenia of 824 cells/uL (reference range, 1500 to 7000 cells/uL), hyperproteinemia of $7.9 \mathrm{~g} / \mathrm{dL}$ (reference range, 6 to $7.8 \mathrm{~g} / \mathrm{dL}$ ), hyperglycemia of 198 $\mathrm{mg} / \mathrm{dL}$ (reference range 56 to $153 \mathrm{mg} / \mathrm{dL}$ ), elevated CK of $7718 \mathrm{mg} / \mathrm{dL}$ (reference range, 55 to $688 \mathrm{mg} / \mathrm{dL}$ ), elevated AST of $99 \mathrm{U} / \mathrm{L}$ (reference range, 14 to $54 \mathrm{U} / \mathrm{L}$ ), and elevated total bilirubin of $0.3 \mathrm{mg} / \mathrm{dL}$ (reference range, 0 to $0.2 \mathrm{mg} / \mathrm{dL}$ ). These findings were most consistent with stress and prolonged muscle rigidity. On urinalysis triple phosphate crystals, moderate rods, and many cocci were present. A urine culture was negative. The cat was placed on intravenous fluid therapy (Lactated Ringers Solution $+20 \mathrm{mEq} \mathrm{KCl} / \mathrm{L}$ at $8 \mathrm{ml} / \mathrm{hr}$ ) and was given a dose of buprenorphine $(0.01 \mathrm{mg} / \mathrm{kg}$ IV once) due to concerns of the cat being painful. About nine hours after admission, the cat had an episode where it became rigid and then limp. The cat became agonal and went into cardiopulmonary arrest. Resuscitation was attempted but was unsuccessful. A postmortem examination was performed. Gross examination revealed chronic, diffuse, mild fibrous visceral adhesions of the gastrointestinal tract and mesentery; mild diffuse pulmonary edema; chronic, multifocal siderofibrotic plaques of the spleen; and a focal nodule within the wall of the mid-urethra. Initial sectioning of the brainstem and cerebellum revealed no gross lesions. Histopathologic examination of the cerebellum revealed diffuse, multifocal, extensive hemorrhages within all layers. Multifocally, small to medium sized blood vessels were occluded by amorphous, glassy, blue-gray emboli that occasionally appeared cellular, reminiscent of chondrocytes (Fig. 1). The material stained positive with Alcian blue, indicative of a cartilaginous matrix (Fig. 2).

Several large meningeal blood vessels, including one on the ventral aspect of the brainstem contained similar emboli. The remainder of the central nervous system was unremarkable. Histopathologic examination of the remainder of the body revealed hepatic, pulmonary, and renal hemorrhages.

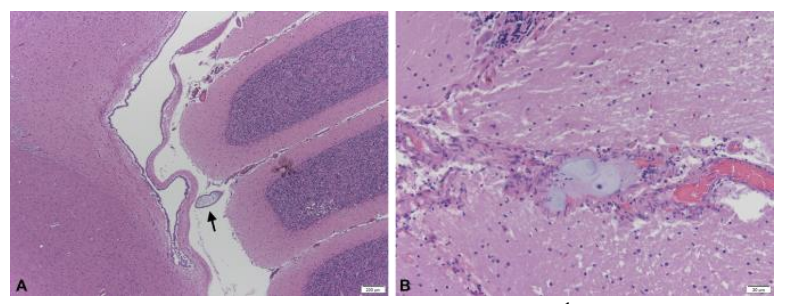

Fig. 1. (A): Cerebellar vermis with $4^{\text {th }}$ ventricle and brainstem. FCE in arteriole (arrow) (20X magnification with H\&E stain). (B): FCE in cerebellar arteriole (200X magnification with $H \& E$ stain).

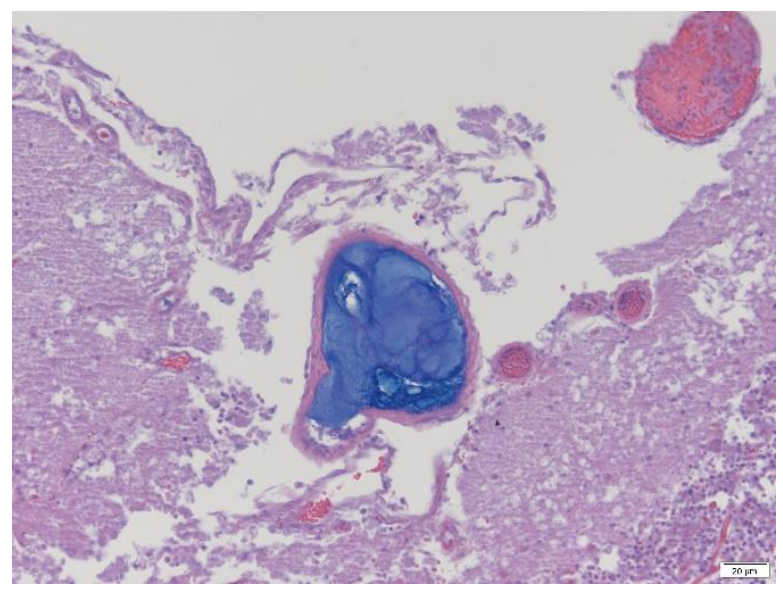

Fig. 2. Evidence of FCE in cerebellar arteriole. Alcian blue stains mucopolysaccharides/hyaline cartilage blue (400X magnification with Alcian blue stain).

The cause of these hemorrhages was unknown though extensive endothelial damage may have triggered disseminated intravascular coagulation. The final histopathological diagnosis was multiple fibrocartilaginous emboli (FCE) with hemorrhage.

\section{Discussion}

Further diagnostics were not performed in this case due to the rapid decline and ultimate death of the patient. However, diagnostics to consider include MRI and cerebrospinal fluid analysis. These diagnostics are not likely to be sensitive or specific to the diagnosis of FCE and findings are likely to be consistent with other vascular-type events.

FCE causing an encephalopathy has been rarely reported in both human and veterinary medicine and has never been documented in a cat. Axlund et al. (2004) reported a case of FCE of the brainstem and midcervical spinal cord in a 4-year old Maltese dog. The dog presented for an acute onset of ataxia and left thoracic limb paralysis.

Neurologic examination findings were suggestive of multifocal disease affecting both the left lateral cervical intumescence and caudal brainstem. The dog showed no clinical improvement following treatment with corticosteroids and neurologically declined. 
The dog was euthanized and a postmortem examination revealed fibrocartilaginous material within the veins of the brainstem and midcervical spinal cord (Axlund et al., 2004).

In addition, a case of FCE causing a multifocal ischemic encephalopathy in a 1-week old, female Suffolk cross Texel lamb has been reported. The lamb presented for generalized tremors but was otherwise neurologically normal.

Post-mortem examination revealed infarction to the medulla, cerebellum, and multiple areas of the spinal cord. Histological analysis revealed evidence of material identical to nucleus pulposus within the vessels (Jeffrey and Wells, 1986).

To date, a total of sixty-seven cases of FCE in humans have been reported; forty-one tissue-confirmed and twenty-six clinically suspected. Of the forty-one tissue confirmed cases, seven showed evidence of extension into the medulla oblongata and one showed evidence of extension into the cerebral arteries (Kepes and Reynard, 1973; Toro-Gonzalez et al., 1993; AbdelRazek et al., 2016).

This is the first case of an FCE causing an encephalopathy in a cat reported to the author's knowledge. Vascular events causing encephalopathies are commonly associated with hypertension secondary to comorbidities in cats and the prognosis and treatment depend on the underlying cause (Boudreau, 2018).

A recent report of feline ischemic myelopathy and encephalopathy in five cats showed evidence of hyaline degenerative arteriopathy within the ventral spinal and/or basilar arteries.

Co-morbidities reported included hypertrophic cardiomyopathy, hyperthyroidism, and chronic renal disease (Rylander et al., 2014). Another case series of eight cats with acute non-progressive neurologic signs showed evidence of ischemic myelopathies and encephalopathies.

Co-morbidities were reported in all but one of these cases and included hypertrophic cardiomyopathy, hyperthyroidism, and chronic renal disease (Simpson $e t$ al., 2014).

Treatment recommendations for FCE induced encephalopathies would likely align with treatment for any vascular-induced encephalopathy and is mostly supportive care such as fluid therapy and physiotherapy. Prognosis is difficult to state with only one case reported. While this is an extremely rare disease, it is an important differential diagnosis that should be included in cases of acute encephalopathies in cats.

\section{Acknowledgments}

The authors would like to acknowledge Jennifer Ginn, DVM for her assistance with the case.

\section{Conflict of interest}

The authors declare that there is no conflict of interest.

\section{References}

AbdelRazek, M.A., Mowla, A., Farooq, S., Silvestri, N., Sawyer, R. and Wolfe, G. 2016. Fibrocartilaginous embolism: a comprehensive review of an under-studied cause of spinal cord infarction and proposed diagnostic criteria. J. Spinal Cord Med. 39, 146-154.

Abramson, C.J., Platt, S.R. and Stedman, N. 2002. Tetraparesis in a cat with fibrocartilaginous emboli. J. Am. Anim. Hosp. Assoc. 38, 153-156.

Axlund, T.W., Isaacs, A.M., Holland, M. and O'Brien, D.P. 2004. Fibrocartilaginous embolic encephalomyelopathy of the brainstem and midcervical spinal cord in a dog. J. Vet. Intern. Med. 18, 765-767.

Barker, E.N., Schofield, E. and Granger, N.P. 2014. What is your neurologic diagnosis? Fibrocartilaginous embolism in a cat. J. Am. Vet. Med. Assoc. 245, 49-51.

Boudreau, C.E. 2018. An update on cerebrovascular disease in dogs and cats. Vet. Clin. North Am. Small Anim. Pract. 48, 45-62.

Cauzinille, L. 2000. Fibrocartilaginous embolism in dogs. Vet. Clin. North Am. Small Anim. Pract. 30, 155-167.

Coradini, M., Johnstone, I., Filippich, L.J. and Armit, S. 2005. Suspected fibrocartilaginous embolism in a cat. Aust. Vet. J. 83, 550-551.

De Risio, L. and Platt, S. 2010. Fibrocartilaginous embolic myelopathy in small animals. Vet. Clin. North Am. Small Anim. Pract. 40, 859-869.

Fuentealba, I.C., Weeks, B.R., Martin, M.T., Joyce, J.R. and Wease, G.S. 1991. Spinal cord ischemic necrosis due to fibrocartilaginous embolism in a horse. J. Vet. Diagn. Invest. 3, 176-179.

Gandini, G. and Cizinauskas, S. 2003. Fibrocartilaginous embolism in 75 dogs: clinical findings and factors influencing recovery rate. J. Small Anim. Pract. 44, 76-80.

Jeffrey, M. and Wells, G.A.H. 1986. Multifocal ischaemic encephalomyelopathy associated with fibrocartilaginous emboli in the lamb. Neuropathol. Appl. Neurobiol. 12, 415-424.

Johnson, R.C., Anderson, W.I. and King, J.M. 1988. Acute pelvic limb paralysis induced by a lumbar fibrocartilaginous embolism in a sow. Cornell Vet. 78, 231-234.

Kepes, J.J. and Reynard, J.D. 1973. Infarction of spinal cord and medulla oblongata caused by fibrocartilaginous emboli: Report of a case. Virchows. Arch. A. Pathol. Pathol. Anat. 361, 185193.

Mikszewski, J.S., Van Winkle, T.J. and Troxel, M.T. 2006. Fibrocartilaginous embolic myelopathy in five cats. J. Am. Anim. Hosp. Assoc. 42, 226-233.

Rylander, H., Eminaga, S., Palus, V., Steinberg, H., 
Caine, A., Summers, B.A., Gehrke, J., West, C., Fox, P.R., Donovan, T. and Cherubini, G.B. 2014. Feline ischemic myelopathy and encephalopathy secondary to hyaline ateriopathy in five cats. J. Feline Med. Surg. 16, 832-839.

Scott, H.W. and O’Leary, M.T. 1996. Fibrocartilaginous embolism in a cat. J. Small Anim. Pract. 37, 228-231.

Simpson, K.M., De Risio, L., Theobald, A., Garosi, L. and Lowrie, M. 2014. Feline ischemic myelopathy with a predilection for the cranial cervical spinal cord in older cats. J. Feline Med. Surg. 16, 10011006.

Stedman, N.L., Brown, T.P. and Rowland, G.N. 1998. Intravascular cartilaginous emboli in the spinal cord of turkeys. Avian Dis. 42, 423-488.

Taylor, H.W., Vandevelde, M. and Firth, E.C. 1977. Ischemic myelopathy caused by fibrocartilaginous in a horse. Vet. Pathol. 14, 479-481.

Toro-Gonzalez, G., Navarro-Roman, L., Roman, G.C., Cantillo, J., Serrano, B., Herrera, M. and Vergara, I. 1993. Acute ischemic stroke from fibrocartilaginous embolism to the middle cerebral artery. Stroke 24, 738-740.

Turner, P.V., Percy, D.H. and Allyson, K. 1995. Fibrocartilaginous embolic myelopathy in a cat. Can. Vet. J. 36, 712-713.

Zaki, F.A., Prata, R.G. and Werner, L.L. 1976. Necrotizing myelopathy in a cat. J. Am. Vet. Med. Assoc. 169, 228-229. 